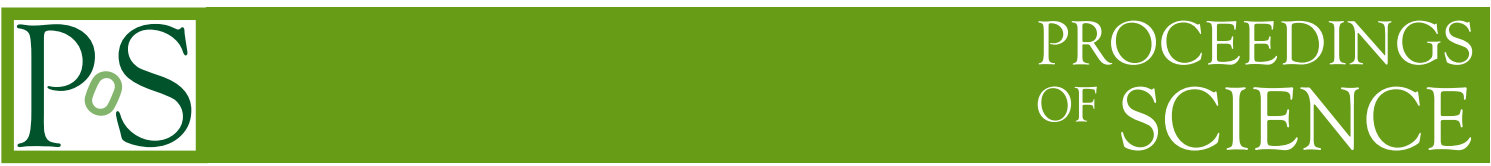

\title{
LHCspin: a polarized internal target for the LHC
}

\section{Paolo Lenisa*}

Universitá di Ferrara and INFN, Italy

E-mail: lenisa@fe.infn.it

\section{Erhard Steffens}

Physics Dept., FAU Erlangen-Nürnberg, Germany

E-mail: erhard.steffensafau.de

\section{Vito Carassiti}

Universitá di Ferrara and INFN, Italy

E-mail: vito@fe.infn.it

\section{Giuseppe Ciullo}

Universitá di Ferrara and INFN, Italy

E-mail: ciullodfe.infn.it

\section{Pasquale Di Nezza}

INFN Laboratori Nazionali di Frascati, Italy

E-mail: pasquale.dinezza@lnf.infn.it

\section{Luciano Libero Pappalardo}

Universitá di Ferrara and INFN, Italy

E-mail: pappalardo@fe.infn.it

\section{Alexander Vasilyev}

PNPI Gatchina, Petersburg, Russia

E-mail: vasilyev_aa@pnpi.nrcki.ru

The LHCspin project aims to bring both unpolarized and polarized physics at the LHC through the installation of a gaseous fixed target at the upstream end of the LHCb detector. The forward geometry of the $\mathrm{LHCb}$ spectrometer $(2<\eta<5)$ is perfectly suited for the reconstruction of particles produced in fixed-target collisions. The fixed-target configuration, with center-of-mass energies ranging from $\sqrt{s_{N N}}=72 \mathrm{GeV}$ in collisions with $\mathrm{Pb}$ beams to $\sqrt{s}=115 \mathrm{GeV}$ in pp interactions, allows to cover a wide backward center-of-mass rapidity region, corresponding to the poorly explored high $\mathrm{x}$-Bjorken and high $\mathrm{x}$-Feynman regimes. The use of transversely polarized $\mathrm{H}$ and $\mathrm{D}$ targets will allow to study the quarks TMDs in p-p collisions at unique kinematic conditions. Furthermore, being LHCb specifically designed for heavy-flavor physics, final states with c- or b-quarks (e.g. inclusive quarkonia production) will be efficiently reconstructed, thus providing, among other fundamental measurememnts, access to the so-far unknown gluons TMDs. The contribution focuses on the design considerations of the polarized internal target and on a discussion of possible critical machine issues.

The 18th International Workshop on Polarized Sources, Targets, and Polarimetry, PSTP2019

23-27 September, 2019

Knoxville, Tennessee

\footnotetext{
*Speaker.
} 


\section{Introduction}

Fixed-target $\mathrm{pp}$ and $\mathrm{pA}$ collisions with a proton beam at the $\mathrm{TeV}$ scale provide unique laboratories for the study of the nucleon?s internal dynamics and, more in general, for the investigation of the complex phenomena arising in the non-perturbative regime of QCD. In particular, due to the substantial boost of the reaction products in the laboratory frame, fixed-target collisions measured with a forward spectrometer such as LHCb, allow one to access the backward center-of-mass rapidity region $\left(-3<y^{*}<0\right)$, corresponding to the poorly explored high $\mathrm{x}$-Bjorken regime. These measurements will thus allow to open the way to innovative and fundamental measurements in regions of the kinematic plane which are still essentially unexplored [1], Fig.1. Furthermore, the use of a gaseous target has the advantage of allowing for a broad variety of nuclear targets, thus providing novel probes for the study of the nucleon and nuclear structure, and for measurements of great interest ranging from heavy-ion physics to cosmic rays physics and dark matter search.

Since 2017, the LHCSpin study group is investigating the installation of a HERMES type polarized gas target (PGT) in front of the LHCb detector. In cooperation with LHC experts, the conditions for applying a PGT are being studied. As a viable option, a cold openable storage cell is considered. A key role for avoiding instabilities of the $7 \mathrm{TeV}$ proton beam is the choice of a proper coating and the suppression of wake fields. A first warm $(300 \mathrm{~K})$ test storage cell is planned for installation in 2020 inside the VELO vessel. It will improve the ongoing SMOG program of LHCb fixed target measurements, and will provide valuable experience of running a storage cell in the harsh LHC environment. The status of the design considerations on a PGT in the LHC beam and of the discussion of critical machine issues is presented.
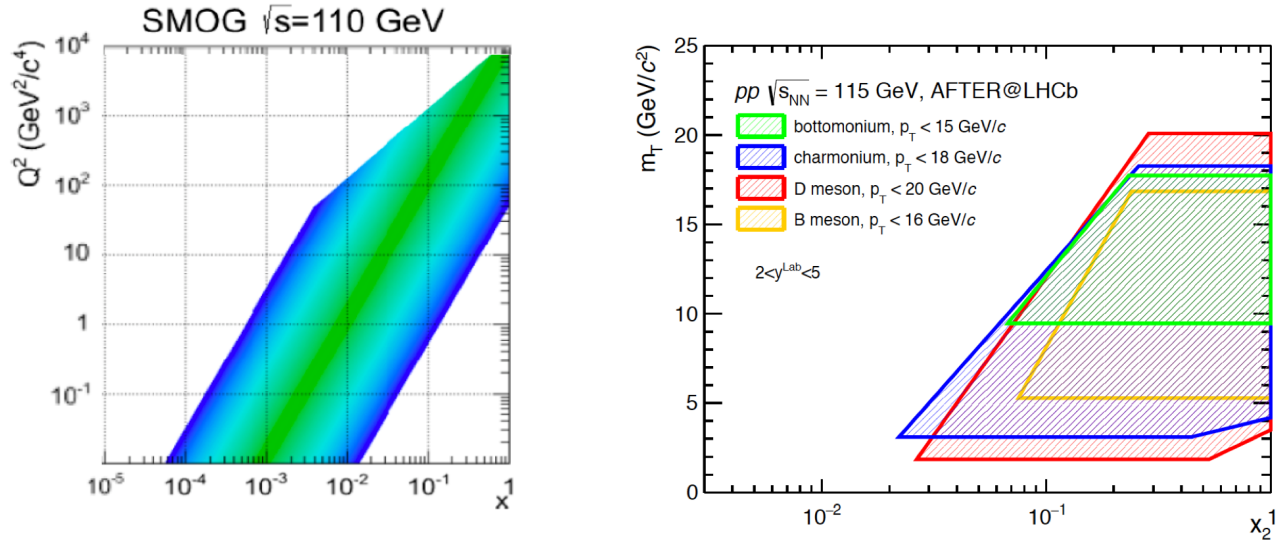

Figure 1: Left: Accessible range in the (x, $\left.Q^{2}\right)$ plane with fixed-target collisions at LHCb (SMOG) [2]. Right: Expected coverage in $\mathrm{x}$-Bjorken for several processes including production of charmionium and bottomonium and D and B mesons within the LHCb acceptance $(2<\eta<5)[1]$

\section{LHCb detector and SMOG upgrade to SMOG2}

The LHCb Spectrometer at the LHC (CERN) detects particles from collisions at IP8. Primary and secondary vertices are reconstructed by means of the VErtex LOcator (VELO) (Fig. 2). The 
luminosity of the colliding bunches is measured via p-e scattering on the residual gas. In order to increase the precision of the luminosity measurements, a gas injection system (SMOG) for $\mathrm{Ne}$ (He, Ar) has been added. Starting in 2015, SMOG has been employed for Fixed-Target (FT) measurements, e.g. that of antiproton production in p-He FT collisions [3]. At present, the LHCSpin group works on the design to improve the SMOG FT measurements by adding a storage cell within the VELO vessel, upstream of the vertex detector. This project is called SMOG2. A design has been presented to the LHCb collaboration in Fall 2018 (installation in February 2020).

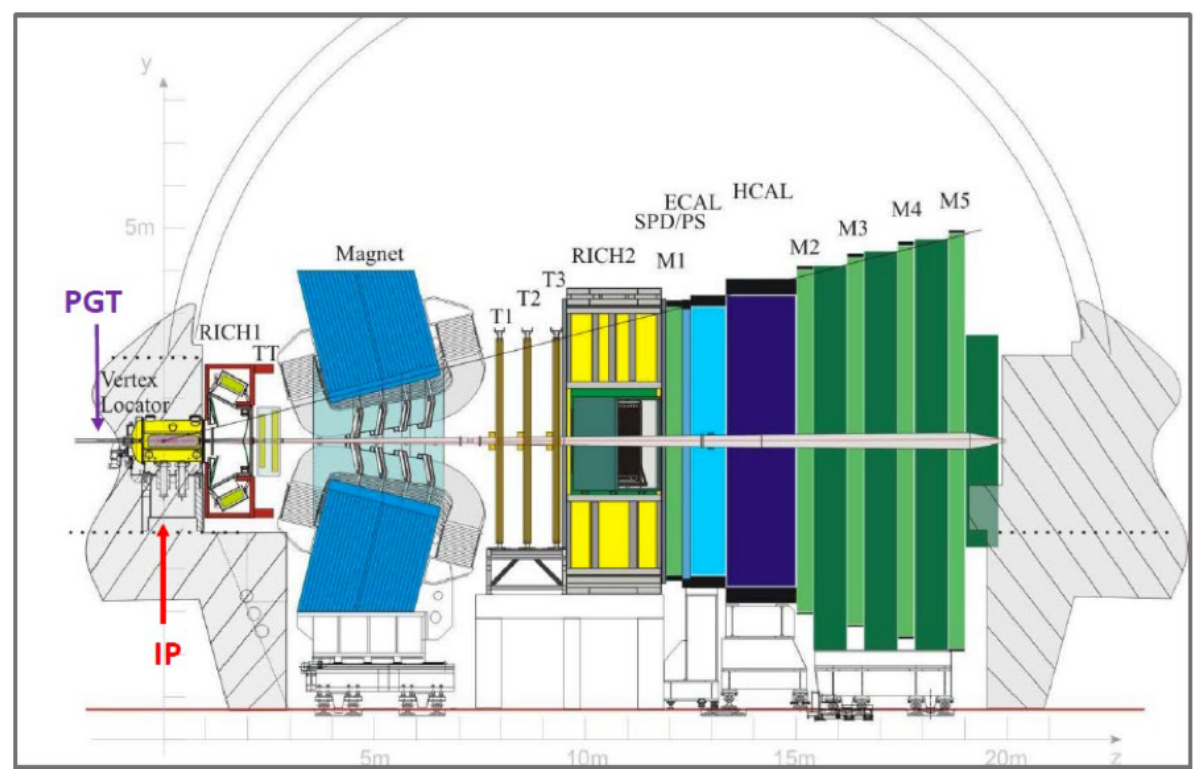

Figure 2: Sideview of the LHCb detector. Left: IP8 with VELO and the possible location of the target (PGT).

The SMOG2 openable target cell (length $\mathrm{L}=20 \mathrm{~cm}$, inner diameter $\mathrm{D}=1.0 \mathrm{~cm}$ ) consists of two halves, connected to the two detector boxes (see Fig. 3). Prior to beam injection the cell is opened with the VELO boxes, and closed during stable operation of the LHC beam, when the VELO detector moves in. The gas is directly injected into the cell center resulting in a triangular pressure bump of length $L$ and central density $\rho_{0}$ in the center [4]. The target areal density $\theta=\rho_{0} \cdot L / 2$ will increase by two orders of magnitude higher with respect to SMOG at the same gas flow rate (see Tab. 1). The cell is complemented by openable conducting surfaces, the Wake Field Suppressors (WFS), with smooth variation of the cross section in order to avoid excitation of wake fields.

The following Table 1 shows the expected SMOG2 target densities for different gases at cell temperature $300 \mathrm{~K}$, and for a typical SMOG flow rate $Q_{0}=1.3 \cdot 10^{-4} \mathrm{mbar} \mathrm{l} / \mathrm{s}$. The scale factor shows the improvement by the storage cell. The strong dependence on the molecular mass $\mathrm{M}$ comes from the $\sqrt{M / T}$ factor of $\theta$, leading to a higher density for the heavier gases.

\subsection{SMOG2 target cell system}

The storage cell system [5] (see Fig. 4 left to right) is composed of the following elements: (i) upstream beam tube, (ii) cylindrical slotted WFS (yellow), (iii) conical WFS and cell tube with wings (blue), (iv) thin contact piece between cell and RF foil (light grey). Elements (ii), (iii) and 


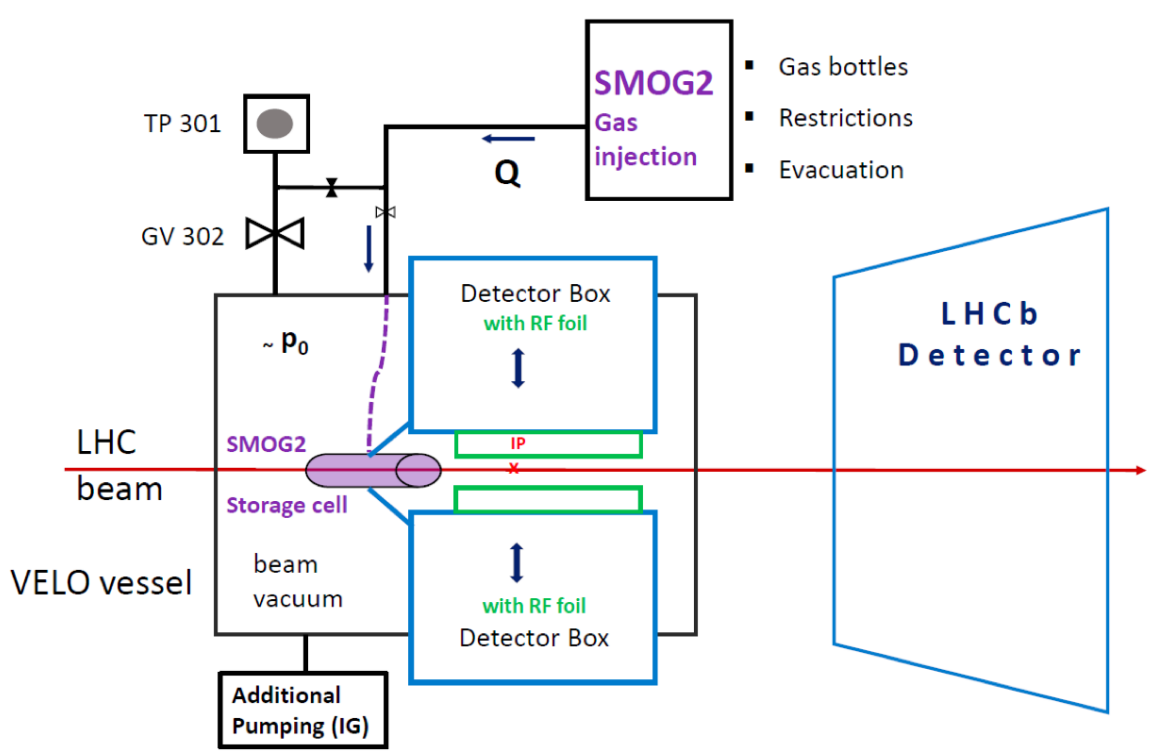

Figure 3: Principle of the SMOG2 target (see text). A gas feed system is employed to inject well-defined flow rates which allow for the precise prediction of the target density.

\begin{tabular}{l|c|c|c|c|c} 
Gas & $\mathrm{H}_{2}$ & $\mathrm{He}$ & $\mathrm{Ne}$ & $\mathrm{Ar}$ & $\mathrm{Kr}$ \\
\hline Areal density $\theta\left(10^{12} \mathrm{~cm}^{-2}\right)$ & 4.3 & 6.0 & 13.4 & 19.0 & 27.4 \\
Scale factor $\left(\theta_{\text {SMOG2 }} / \theta_{\text {SMOG }}\right)$ & 7.6 & 10.7 & 24 & 34 & 49
\end{tabular}

Table 1: SMOG2 target densities for different gases at cell temperature $300 \mathrm{~K}$ a flow rate of $Q_{0}=1.3 \cdot 10^{-4}$ mbar $1 / \mathrm{s}$.

(iv) consist of two halves and open with the detector boxes (dark grey). The cell system is made of $\mathrm{Al}(99.5 \%)$ for the rigid parts (iii), and of $\mathrm{Cu}-\mathrm{Be}$ for the flexible parts (ii), (iv). In the cell center, a small capillary for gas injection is visible. In the open position of the cell system, a free space of $\geq 50 \mathrm{~mm}$ in diameter is provided during injection and tuning of the beam. The cell is suspended by curved light-weight bars connected to the detector boxes, and by the contact pieces (iv) that fix the cell in the lateral degree of freedom. One cell halve is rigidly connected to the box, while the opposite halve can slightly move during closing, being engaged by the rigid one and thus fixed to the axis of the VELO detector. All surfaces seen by the beam must be covered with a low Secondary Electron Emission (SEY) coating, like NEG or amorphous Carbon (a-C).

\section{Polarized Target for the LHC}

In parallel to the SMOG2 project, a conceptual design is being studied, investigating critical machine issues which may represent a show stopper for operating a polarized gas target in the LHC. Such items are:

- a cell system of conductive surfaces with low SEY to prevent instabilities by the formation of electron clouds (EC); 


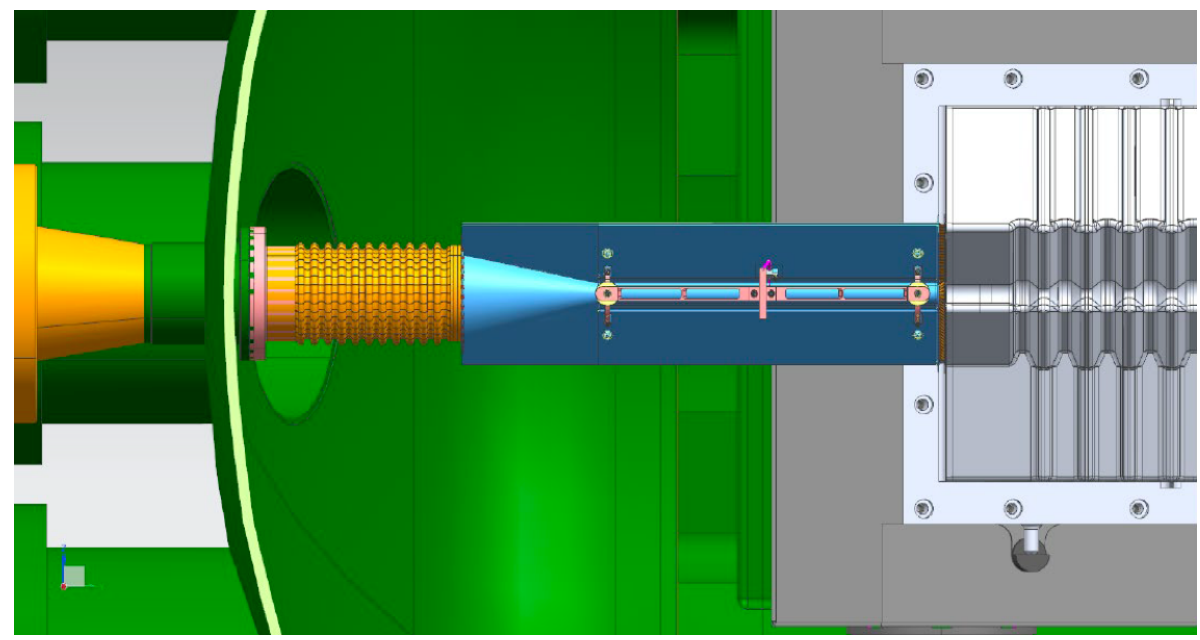

Figure 4: Side view of the SMOG2 storage cell system (see text). The beam employed for FT measurements is directed from left to right. The secondary particles move to the right into the VELO detector (light grey).

- a cell surface with low recombination to avoid polarization losses, in accordance with the restrictions imposed by a safe operation of the LHC;

- a target chamber with transverse guide field, an openable cell system and an effective differential pumping of the target gas;

- optimum conditions for low Beam-Induced Depolarization (BID) of the target atoms by the periodic bunch fields.

\subsection{Suppressing of beam instabilities}

The cell system consisting of conductive surfaces may cause instabilities of two kinds: (a) by its shape which will determine its RF properties, e.g rapidly changing cross section or cavity-like structures, or (b) by surfaces with high yield of secondary electrons (high SEY) causing dense electron clouds trapped by the positive beam charge.

(a) By the bunch fields, excitation of excessive impedances in frequency space may occur, leading to energy dissipation in the surrounding chambers (beam induced heating), or to resonant kicks to the following bunches, resulting in beam blow up and losses. These effects can cause severe damage to the machine and its components, in particular at the LHC with its huge peak power where sophisticated collimator and beam dump systems are needed to protect the machine. All parts to be installed into the LHC are carefully inspected and approved by the Impedance group using numerical simulations and RF measurements. The SMOG2 target system shown in Fig. 4 has already been inspected [6] and approved as outlined in [5].

(b) The formation of dense Electron Clouds (EC) may lead to transverse oscillations of the beam with exponential growth, resulting in dangerous beam losses [6]. Evidently, the elevated residual gas pressure in the target region may favour EC formation, as well as chamber walls with high SEY. As coatings for surfaces close to the LHC beam, materials with SEY $\leq 1.4$ are allowed, only. These are (i) Non-Evaporable Getter (NEG) and (ii) amorphous Carbon (a-C). NEG coating for the tube's inner surface is excluded because of its pumping action which will result 
in embrittlement and possible disintegration of the coating. At present, amorphous Carbon (a-C) seems to be the only viable solution. A-C is already applied in accelerators, including the SPS and the LHC. For coating beam screens of accelerator magnets, it can even be applied in situ, without removing the magnets.

\subsection{Beam-induced depolarization}

Beam Induced Depolarization (BID) is based on resonant transitions caused by the beam field acting on the polarized $\mathrm{H}$-atoms in an external strong guide field $B_{0}(\approx 300 \mathrm{mT})$ (Fig. 5).
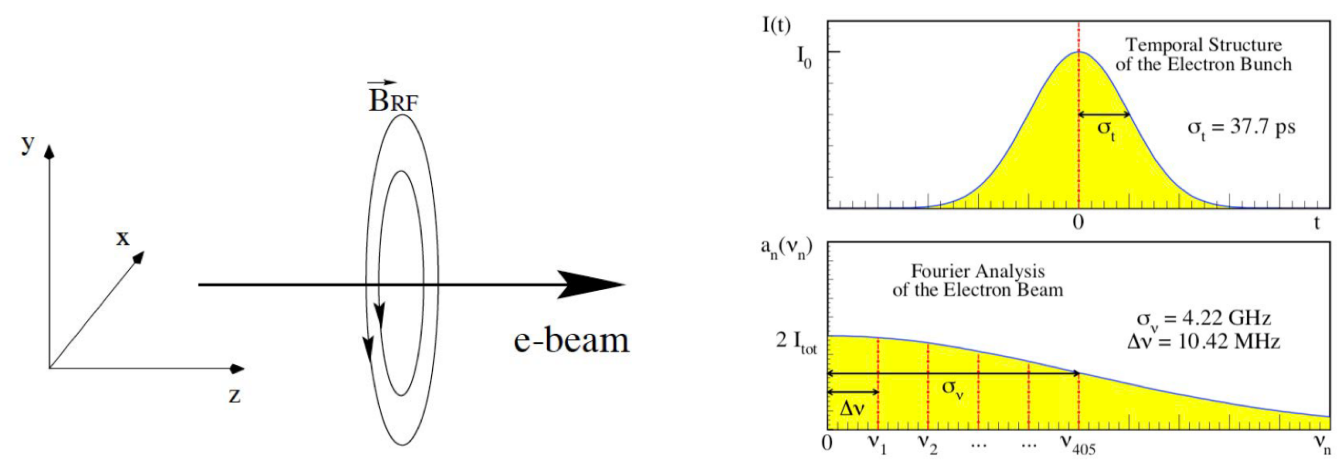

Figure 5: Left: magnetic RF-field associated with a bunched e-beam. Right: time structure and corresponding Fourier composition of the LHC bunched beam.

There are different classes of transitions, depending on the relative orientation of the guide field $B_{0}$ and $B_{1}$, the component of the beam field. With total spin F (for the notation see Fig. 6 and Ref. [4]) one has two classes of resonances:

- $\pi$ resonances for $B_{1} \perp B_{0}$ with selection rule $\Delta F=0, \pm 1$ and $\Delta m_{F}= \pm 1$

- $\sigma$ resonances for $B_{1} \| B_{0}$ with selection rule $\Delta F= \pm 1$ and $\Delta m_{F}=0$.

Depolarization of the HERMES Polarized Gas Target [7] by the HERA $e^{ \pm}$beams has been discussed in the 1989 proposal and provisions for high target polarization were taken in the course of the target development. The experimental study with longitudinal guide field $B_{\|}$and optimization is described in Ref. [8]. Additional measurements with transverse $B_{\perp}$ together with the analysis of all measurements were subject of a dissertation by Phil Tait (Erlangen 2006) [9]. Its application to the LHC is discussed in a recent report [10].

Some of these resonances change nuclear polarization. For longitudinal guide field, only the $\pi$ resonances are present. For transverse field, like for HERMES run 2 or at LHCSpin, both types of resonances are present. The $\sigma$ resonances, interchanging states 2 and 4, are densely spaced, i.e. its prevention requires a very high homogeneity of the guide field. At HERMES during transverse running (2001-2004), careful studies to optimize the target polarization have been performed. As diagnostic tool, the target polarimeter with its capability of detecting single substates has been used. During the next LHC running period, a beam current of about $1 \mathrm{~A}$ is envisaged, which is 25 times higher than at HERAe. A first attempt of a preliminary comparison is shown in a CERN-PBC note [10]. The relevant beam parameters of HERA-e and LHC are shown in Table 2. The main differences between HERA-e and LHC are: 

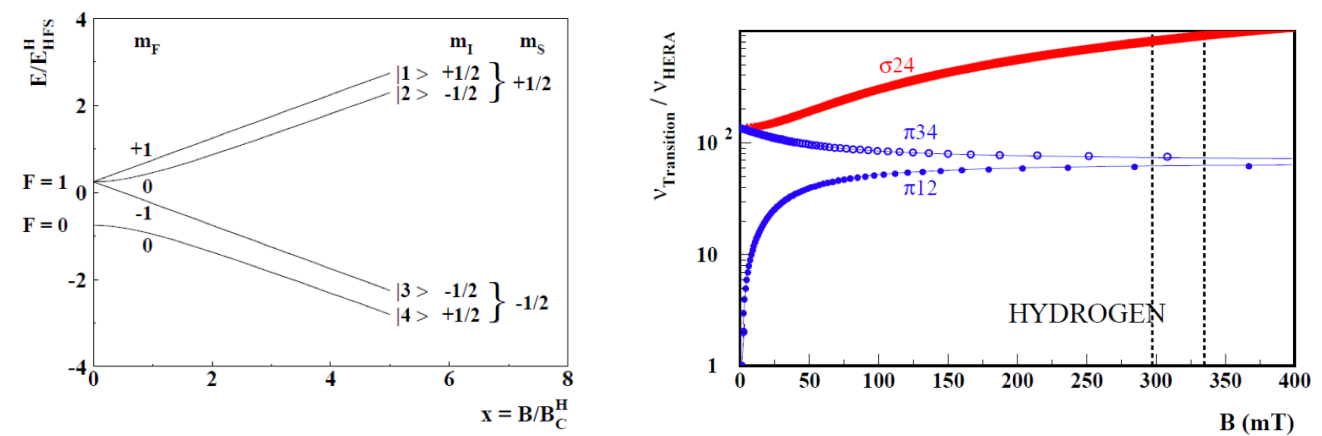

Figure 6: Left: hyperfine state diagram of hydrogen. The energy of the substates is shown as function of the external B-field in units of $B_{c}=50.7 \mathrm{mT}$. F = total spin; I, s = nuclear and electron spin. The substates are denoted by $|1>-| 4>$ with decreasing energy. Right: $\sigma$ and $\pi$ transitions. The vertical axis refers to a renormalization with the revolution frequency of the beam at HERA. The plot evidences the close energyspacing of the $\sigma$ transitions.

\begin{tabular}{l|c|c|c|c|c} 
Machine & $N_{\text {bunch }}$ & $f_{\text {bunch }}(\mathrm{MHz})$ & $I_{\text {beam }}(\mathrm{A})$ & $\sigma_{t}(\mathrm{ps})$ & $1 / \mathrm{e}-$ Fourier spectrum \\
\hline HERA-e & 210 & 10.41 & 0.04 & 31 & $5.1 \mathrm{GHz}$ \\
LHC & 2600 & 40.08 & 1.0 & 253 & $0.63 \mathrm{GHz}$
\end{tabular}

Table 2: Relevant beam parameters of HERA-e and LHC

- Bunch frequency $f_{B}: 10.41 \mathrm{MHz}$ vs. $40.08 \mathrm{MHz}$. This results for the LHC in a 4 times larger spacing of the resonant $B$-values, i.e. it lowers the requirements on field quality.

- Width of the Fourier spectrum: $5.1 \mathrm{GHz}$ vs. $0.63 \mathrm{GHz}$. This leads to a rapid fall-off of the relevant Fourier amplitudes of the $\sigma_{2-4}$ resonance $(8.54 \mathrm{GHz})$ at the LHC.

This naïve comparison suggests that BID at the LHC is probably less dangerous as at HERA-e, thanks to the higher bunch frequency and the much longer bunches, and despite the 25 times higher beam current. Clearly, the estimation has to be confirmed by a systematic study of BID at the LHC.

\section{Arrangement in the tunnel}

The PGT has to fit into the space upstream of the VELO vessel up to a shielding wall, which leaves about 1 meter for the target (Fig. 7). Moving the wall would give more space, but it is important that the PGT stays in the acceptance of the VELO detector. The minimum distance to the VELO is given by considerations on effective differential pumping of the peaked flow from the downstream cell end. In the horizontal transverse direction, there is enough space to place the Atomic Beam Source (ABS) and diagnostics in the horizontal plane, which implies a vertical guide field $B_{0}$.

\section{Vacuum system and estimate of the target density}

The arrangement of the PGT in the beam line upstream of the VELO is shown schematically in Fig. 8. An intermediate configuration for Run 3 including a valve for sectoring the LHC vacuum 


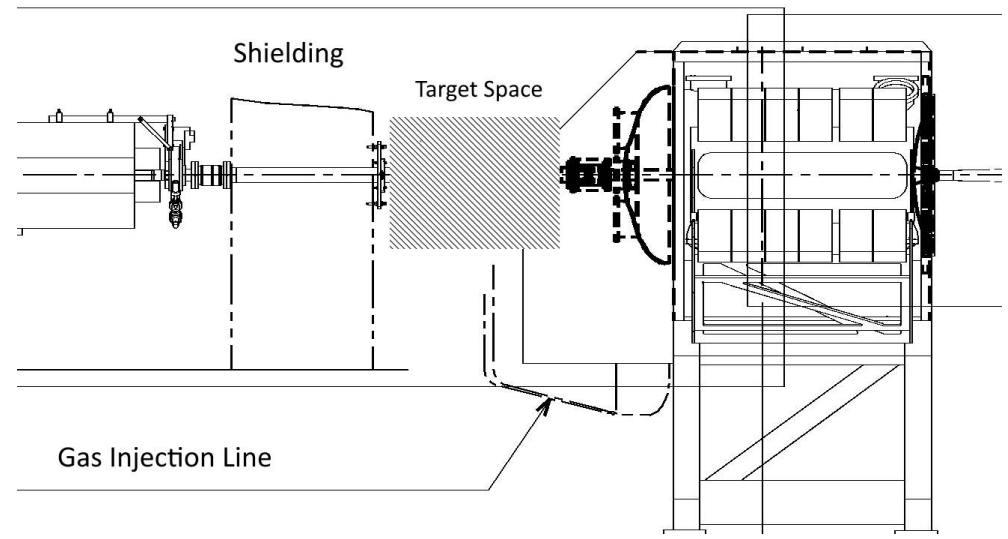

Figure 7: View of the region upstream the VELO detector evidencing the space available for the PGT.

is shown which allows for the installation of fixed-target experiments upstream of VELO without breaking the vacuum in the VELO vessel. The PGT cannot be located close to or even inside the VELO vessel because of the high gas flow of atomic and recombined hydrogen which requires differential pumping on a separate target chamber.

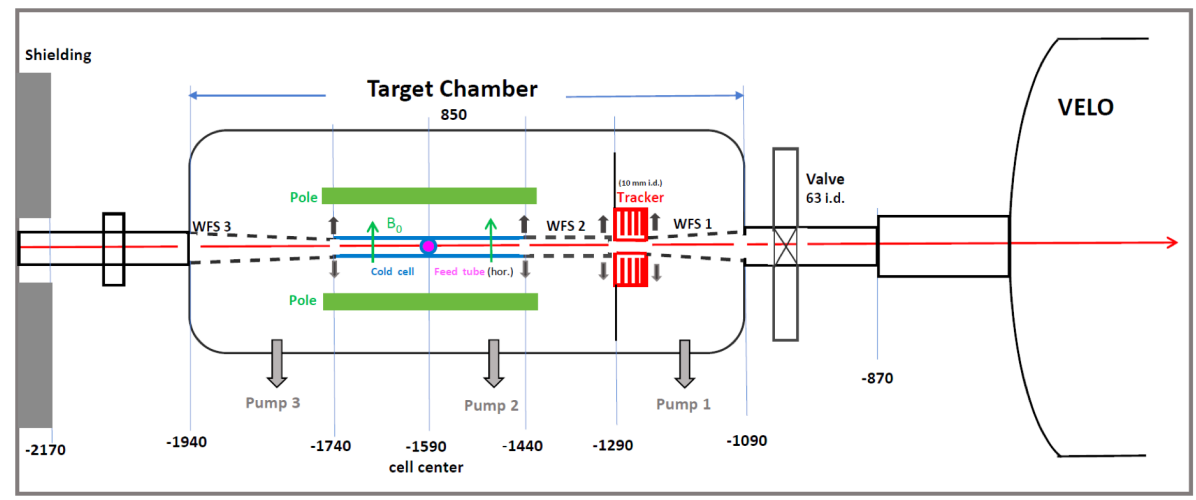

Figure 8: The conceptual drawing shows a sideview of the proposed PGT arrangement upstream of VELO with (right to left): new sectoring valve (installed in 2020), target chamber with conical WFS1 ( $\mathrm{L}=20 \mathrm{~cm}$ ), Tracker (red) with $1 \mathrm{~cm}$ opening, gridded tube WFS $2(\mathrm{~L}=15 \mathrm{~cm})$ for differential pumping, cold cell $(\mathrm{L}=$ $30 \mathrm{~cm}$ ) with beam tube and feed tube for injection of ABS beam in a vertical guide field $B_{0}$ of about $0.3 \mathrm{~T}$. The different pumping stages are indicated. The openable components are denoted with black arrows. The approximate positions are denoted by $\mathrm{z}$ (in $\mathrm{mm}$ ). The IP8 is within the VELO detector at $\mathrm{z}=0$.

An estimate of the peaked flow out of the downstream cell opening shows that for the dimensions assumed, about $4 \%$ of the down-stream flow passes the $1 \mathrm{~cm}$ opening in the Tracker, $30 \mathrm{~cm}$ downstream of the cell center. For the full ABS flow, this would correspond to about $10^{-6} \mathrm{mb}$ 1/s of recombined $\mathrm{H}_{2}$. This is within the tolerable range. The target density is estimated with the following assumptions:

- $I_{H}(100 \%$ HERMES ABS flow $)=6.5 \cdot 10^{16} / \mathrm{s}$, may be limited by LHC vacuum constraints or space limitations for the PGT; cell $30 \mathrm{~cm}$ long, $1.0 \mathrm{~cm}$ inner diameter (i.d.), at 100K, with standard feed tube $10 \mathrm{~cm}$ long, $1.0 \mathrm{~cm}$ i.d. 
- The resulting $100 \%$ density of the polarized gas target is $\theta=1.2 \cdot 10^{14} / \mathrm{cm}^{2}$, comparable with HERMES. For the future HL-LHC-25ns, the maximum luminosity achievable with the PGT would be up to $8.3 \cdot 10^{32} / \mathrm{cm}^{2}$ s. To which extent such densities can be realized and exploited in a real experiment, depends on many factors. This has to be investigated in more detail.

\section{Conclusions}

Fixed target collisions at the LHC offer a unique opportunity for a laboratory for QCD and astroparticle in unexplored kinematic regions. $\mathrm{LHCb}$ is the most suitable experiment able to open this new field of research, also due to the previous experience with the SMOG system. The upgraded unpolarized gas target, SMOG2 will be installed in Spring 2020.

The polarized target is the natural evolution of SMOG2. LHCSpin aims at installing a polarized gas target in front of the LHCb spectrometer, bringing for the first time polarization physics to the LHC. LHCb will then become the first experiment simultaneously running in collider and fixed-target mode with polarized targets, opening a whole new range of explorations to its exceptional spectrometer. The strong interest and support from the international theory community, together with the established experience of the experimental groups involved, will bring forward our knowledge of spin physics and QCD in a broad range of areas to an unprecedented level of sophistication.

The key element, a target chamber with cooled cell to open, differential pumping and tracking detector, has highest priority. If built and tested during Run3 (2021-23), it could be installed in LS3 and tested using a gas feed system. Installation of the other PGT components during Technical Stops appears feasible. The key parameters of the LHCSpin target are promising, as well as the broad physics potential. With enough support from our Community it could become a success!

\section{Acknowledgments}

The project is supported by European Project STRONG - 2020 Grant Agreement no. 824093. The authors are indebted to P. Chiggiato, R. Cimino, M. Contalbrigo, R. Engels, M. Ferro-Luzzi, W. Funk, G. Graziani, K. Grigoryev, G. Iadarola, A. Nass, F. Rathmann, D. Reggiani, B. Salvant and $\mathrm{E}$. Thomas for discussions and valuable advice.

\section{References}

[1] C. Hadjidakis at al., A Fixed-Target Programme at the LHC: Physics Case and Projected Performances for Heavy-Ion, Hadron, Spin and Astroparticle Studies, arXiv:1807.00603 [hep-ex]

[2] PBC Report, Physics opportunities with the fixed target program of the LHCb experiment using an unpolarized gas target, LHCb-PUB-2018-015.

[3] LHCb Collaboration, Measurement of antiproton production in p-He collisions at $\sqrt{s}_{N N}=110 \mathrm{GeV}$ Phys. Rev. Lett. 121 (2018) 22.

[4] E. Steffens \& W. Haeberli, Polarized gas targets, Rep. Prog. Phys. 66 (2003) 1887.

[5] SMOG2 EDR - CERN-PBC-Notes-2018-007. Geneva, CERN, 2018 
[6] B.K. Popovic: SMOG2 preliminary checks for transverse impedance, see: https://indico.cern.ch/event/765714/contributions/

[7] A. Airapetian et al. (HERMES Collaboration): The HERMES Polarized Hydrogen and Deuterium Internal Gas Target, Nucl. Instr. and Meth. A540 (2005) 68.

[8] A. Airapetian et al. (HERMES Collaboration): Beam-induced nuclear depolarization in a gaseous polarized hydrogen target, Phys. Rev. Lett. 82 (1999) 1164.

[9] P. Tait: Beam-Induced Depolarization at the HERMES Transversely Polarized Hydrogen Target, Thesis, Univ. of Erlangen (2006); http://hermes.desy.de/notes/pub/06-LIB/pntait.06-060.thesis.ps.gz

[10] E. Steffens: Beam-Induced Depolarization and Application to a Polarized Gas Target in the LHC beam, CERN-PBC-Note-2018-001. 